\title{
¿Por qué las políticas culturales locales no cambian? Constricciones del modelo urbano, inercia en la gestión y batallas culturales en los «gobiernos del cambio» en Madrid y Barcelona (2015-2018)
}

\author{
Why do local cultural policies not change? Urban model constraints, \\ management inertia and cultural battles in the "governments of change» \\ in Madrid and Barcelona, 2015-18
}

\section{JOAQUIM RIUS-ULLDEMOLINS}

Universitat de València

VERÒNICA GISBERT

Universitat de València

\section{Como citar/Citation}

Rius-Ulldemolins, J. y Gisbert, V. (2018). ¿̨Por qué las políticas culturales locales no cambian? Constricciones del modelo urbano, inercia en la gestión y batallas culturales en los "gobiernos del cambio» en Madrid y Barcelona (2015-2018). Revista Española de Ciencia Política, 47, 93-122. Doi: https://doi.org/10.21308/recp.47.04

\section{Resumen}

Desde los años ochenta, la política cultural ha sido progresivamente entendida como una herramienta para la transformación urbanística y socioeconómica de las ciudades. En este sentido, una creciente atención se ha dirigido a explicar cómo la acción pública en el ámbito cultural ha cambiado en función de los objetivos y de los discursos que la legitiman. Sin embargo, menos atención ha recibido la comprensión de los elementos que explican su continuidad y persistencia, las inercias y las resistencias al cambio de esta política pública. Asimismo, en la última década, la creciente crítica al paradigma de la ciudad creativa y a sus efectos negativos ha llevado a desarrollar una propuesta alternativa de política cultural, en el marco de la llamada «nueva política» heredera del movimiento 15M. Esta perspectiva pretende impulsar nuevas formas de entender la cultura en el ámbito local, que se ha calificado de procomún. Este artículo trata de responder a la pregunta sobre hasta qué punto se puede hablar de cambio o, por el contrario, de continuidad en el desarrollo de las políticas culturales locales en dos casos destacados donde este fenómeno ha ocurrido: Madrid y Barcelona entre 2015 y 2018. También analiza las razones estructurales de la relativa continuidad en 
formas y resultados de la política cultural, a pesar de un cambio discursivo en relación con sus objetivos.

Palabras clave: política cultural, ciudad creativa, campo político, nueva política, Madrid, Barcelona.

\begin{abstract}
Since the 1980s, cultural policy has been progressively understood as a tool for the urban and socio-economic transformation of cities. In this sense, an increasing attention has been addressed at explaining how public action in the cultural sphere has changed according to the objectives and discourses that legitimize it. However, less attention has been paid to understanding the elements that explain its continuity and persistence, inertias and resistances to change of this public policy. Likewise, in the last decade, the growing criticism of the creative city paradigm and its negative effects has led to develop an alternative proposal of cultural policy within the framework of the so-called «new politics» inherited from the $15 \mathrm{M}$ movement. This perspective tries to encourage new ways of understanding culture in the local domain, which has been described as pro-common. This article seeks to give an answer to the question about the extent to which there has been a change or, instead, continuity in the development local cultural policies in two relevant cases where this phenomenon has occurred, Madrid and Barcelona between 2015 and 2018. Moreover, it analyzes the structural reasons for the relative continuity in forms and results of the cultural policy despite a discursive change regarding its objectives.
\end{abstract}

Keywords: cultural policy, creative city, political field, new politics, Madrid, Barcelona

\title{
INTRODUCCIÓN
}

¿Por qué las políticas culturales locales no cambian o lo hacen de una forma muy lenta? O más concretamente, ¿por qué cuesta tanto reorientarlas a partir de un cambio de gobierno y de programas políticos? Estas preguntas son pertinentes en varios contextos, pero especialmente en España, donde las elecciones locales del 2015 representaron un momento de cambio de tendencia en el campo político, con la victoria en una parte significativa de las principales ciudades de candidaturas ciudadanas o coaliciones que en cierta medida eran o se reclamaban herederas del $15 \mathrm{M}$ y apelaban a un cambio de objetivos políticos, de formas de gestión política y hasta de régimen político (Rodon and Hierro, 2016). Un cambio que expresaba los efectos económicos, la crisis de régimen político y la emergencia de una nueva generación con diferentes concepciones acerca de la política y la cultura (Antentas, 2017; Sola y Rendueles, 2018). En este contexto, activistas o académicos han tomado un papel protagonista en los nuevos gobiernos locales de estas ciudades, proponiendo un giro radical en las políticas culturales (Barcelona en Comú, 2015).

Estas demandas de cambio no eran fruto de un capricho momentáneo o de un discurso vacío, sino que partían de un diagnóstico crecientemente compartido acerca de 
los límites y las disfunciones del modelo de la ciudad creativa adoptado en diferentes acentos y modalidades por las grandes ciudades españolas (Rius-Ulldemolins et al., 2015; Rubio y Rius-Ulldemolins, 2015).

Sin embargo, dos años después se empieza a ver un cierto agotamiento de este plan de cambio o un cierto estancamiento en la capacidad de modificar no solo discursivamente la legitimación de las políticas culturales, sino la gestión práctica de sus instituciones y programas de actuaciones. Si los nuevos objetivos de las políticas culturales estaban claros, ¿por qué no se han podido llevar a práctica? ¿Es explicable esta continuidad por la flaqueza conceptual o contradicciones discursivas o bien debemos analizar factores relativos a la base infraestructural de las políticas culturales o a las relaciones de poder en las que se insertan? Este agotamiento del cambio es un aspecto que, si bien empieza a ser debatido en la esfera periodística — especialmente en medios digitales - o activista (Gil, 2017), y ya han empezado a formularse valiosas contribuciones al debate sobre la gestión cultural en los ayuntamientos del cambio (Barbieri, 2018), aún no se ha analizado ni interpretado de manera profunda y basada en un trabajo de campo, como intentaremos hacer en este artículo.

En general, la explicación del cambio histórico y político ha sido objeto central de las ciencias sociales, en un campo en el que los enfoques ideológicos influyen en la misma concepción o posibilidad del cambio (Sztompka, 1993). En cierta medida, la concepción de la política cultural, en su vertiente académica y cercana a la ciencia política (Gray, 2010), ha concebido el desarrollo de la política cultural como algo positivo, con un enfoque próximo al evolucionismo y a la idea de progreso, y ha generado una dinámica inflacionaria en los objetivos y los medios empleada en ella (Urfalino, 1984). Sin embargo, la percepción de su cara oscura o los efectos no deseados (Rius-Ulldemolins et al., 2017), y un enfoque más crítico fundado en los estudios culturales, se ha desarrollado desde los años ochenta (Gray, 2010). Este enfoque ha entroncado con los debates sobre los efectos segregadores o elitistas de las políticas culturales desarrollado por los estudios urbanos (Peck, 2005; Martí-Costa y Pradel i Miquel, 2012).

Asimismo, el cambio en las políticas culturales ha sido previamente estudiado por algunos autores en sus causas sobre todo exógenas y en relación con el campo político (Shockley, 2011; Barbieri, 2012; Barbieri, 2015). Sin embargo, en menor medida han sido abordadas en sus factores endógenos y en la lógica de la relación con el campo cultural y la gobernanza local (Saint-Pierre, 2003; Gray, 2008), que es el factor en el que se ahondará en este artículo. Además, a partir de la concepción de la política cultural como sistema de relaciones y dependencias en el marco local (Rius-Ulldemolins y Martinez Illa, 2016; Patricio Mulero y Rius-Ulldemolins, 2017), este artículo pretende estudiar los agentes y factores que explican el porqué de la inercia y la continuidad, en el marco de unos factores políticos, al menos en apariencia, inestables y cambiantes.

Los casos de Madrid y Barcelona son relevantes para debatir esta cuestión, por su interés como objeto de estudio del cambio político y el cambio en las políticas culturales. Se trata de las dos mayores ciudades del Estado espańol, con una gran presencia 
de sectores e industrias culturales (Méndez et al., 2012), y que han desarrollado dos modelos de política cultural diferenciados. Sin embargo, en ambos casos este ámbito de gestión pública (y su importancia en la economía y la gobernanza local) ha aumentado considerablemente las dos últimas décadas (Rius-Ulldemolins y Sánchez, 2015).

De la misma forma, en ambos casos se produjo un cambio en el gobierno municipal en los comicios celebrados en 2015, en los que dos candidaturas de la llamada nueva política heredera del $15 \mathrm{M}$ se hicieron con las riendas del gobierno local (Antentas, 2017). En este contexto, se generó un debate sobre la necesidad del cambio del modelo de política cultural en el marco del cambio social y urbano. Estas circunstancias y características convierten los dos casos en relevantes y su comparación en una herramienta para contrastar las hipótesis sobre el cambio en la política cultural local. Para desarrollar este análisis, se ha procedido a analizar el contexto político-administrativo y el desarrollo del modelo local de la política cultural, los objetivos marcados por los gobiernos del cambio y su relación con factores y agentes exógenos como son el sistema político y los agentes de los sectores culturales. Posteriormente, se han elegido las dimensiones más significativas de sus factores endógenos, como la forma de gestión y de las prioridades presupuestarias ${ }^{1}$.

El análisis de este artículo está basado en la combinación de diferentes fuentes: en primer lugar, el trabajo realizado en una investigación sobre la financiación y la evolución de las políticas culturales en el Estado español financiada por la Fundación Alternativas en la que se combinó una metodología de análisis de los presupuestos y la realización de grupos de discusión (Rubio Arostegui et al., 2014)². En segundo lugar,

1. Evidentemente, no se pretende en este artículo agotar todas las dimensiones de análisis que podrían incluir un estudio de subcasos referidos a experiencias de autogestión o políticas sectoriales concretes como bibliotecas, teatro, etc., pero entendemos que se consigue trazar un primer balance acerca de la posibilidad y alcance del cambio en la política cultural en este periodo.

2. En el marco de este proyecto se realizaron en 2014 dos grupos de discusión, uno en Madrid y otro en Barcelona, para debatir acerca de las prioridades y la financiación de la política cultural. En ellos participaron los siguientes expertos y agentes clave sobre la política y la gestión cultural: Fátima Anllo (Universidad Complutense de Madrid), Antonio Ariño (Universitat de València), Antonio Ávila Álvarez (Federación de Gremios de Editores de España), Jordi Baltà (Interarts), Lluis Bonet (Universitat de Barcelona), Javier Brun Gonzalez (Ayuntamiento de Huesca), Ruben Caravaca (Asociación de Gestores Culturales de la Comunidad de Madrid), Jorge Fernández León (Ayuntamiento de Gijón), Juan Antonio Hormigón Blánquez (Asociación de Directores de Escena de España), Àngel Mestres (Trànsit Projectes), Jordi Pascual Pascual (UGLC-Agenda 21 de la cultura), Cristina Ramírez (Ayuntamiento de San Fernando de Henares), César Rendueles (Universidad Complutense de Madrid), Francisco Rodríguez Peinado (Asociación de Autores de Cómic de España), Yolanda Romero Gomez (Asociación de Directores de Arte Contemporáneo en España), Joan Subirats (Universitat Autònoma de Barcelona), Juan Alberto Torres Larumbe (Federación Estatal de Compañías y Empresas Profesionales de Danza) y Fernando Victoria de Lecea (Iberrota Films). Aunque los objetos de estudio no fueron exclusivamente Madrid y Barcelona, estos dos casos, por su relevancia, fueron objeto de debate y forman parte del análisis de caso en sus bases y desarrollos anteriores al cambio en 2015. 
esta investigación ha sido actualizada a partir del análisis de contenido de los programas de gobierno, las memorias de realizaciones y los datos presupuestarios y de ejecución en Madrid y en Barcelona. En el caso de Madrid hemos consultado los datos proporcionados por el Anuario Estadístico y los mapas estratégicos del Ayuntamiento de Madrid, portal de transparencia de la empresa municipal Madrid Destino, empresa mixta Club de Campo Villa de Madrid y Ayuntamiento de Madrid y la web del Observatorio de la Ciudad ${ }^{3}$. En relación con el caso de Barcelona se han consultado y analizado los datos proporcionados por la Gaceta Municipal, el Anuario Estadístico del Ajuntament de Barcelona, las Memorias del Instituto de Cultura de Barcelona y la página web del Observatorio de Datos Culturales de Barcelona ${ }^{4}$.

El objetivo de este artículo es analizar la compleja y no siempre evidente relación entre cambio político y cambio en las políticas culturales locales. Para ello, en primer lugar, tomaremos en consideración las transformaciones de las políticas culturales y sus objetivos, debatiendo los factores y agentes que explican los cambios y proponiendo una nueva perspectiva al respecto. A continuación, examinaremos los casos de Madrid y Barcelona entre 2015 y 2018 — los años en que se produce la entrada de los llamados «Ayuntamientos del cambio»-, analizando en profundidad los cambios (o la ausencia de ellos) en la política cultural local y contrastando con ello nuestra hipótesis de los factores que los explican. Finalmente, en las conclusiones realizaremos un breve balance comparando los dos casos y avanzando los límites y perspectivas de los hallazgos para posteriores investigaciones respecto al cambio político y el cambio de las políticas culturales.

\section{POLÍTICAS CULTURALES, CAMBIO POLÍTICO Y CAMBIO SOCIAL}

Después de su invención en los años cincuenta, las políticas culturales se han consolidado como un ámbito cada vez más significativo de la intervención pública, primero en los países avanzados (Urfalino, 1996) y posteriormente en países en vías de desarrollo (Bustamante, 2015). Esta creciente centralidad se explica en parte por la mayor relevancia económica de las industrias creativas y de la dimensión cultural y simbólica de los productos, especialmente aquellos que se asocian con la marca territorial (Scott, 2010; Williams y Currid-Halkett, 2011). De este modo, la creciente importancia del turismo en general y del turismo cultural en particular para las grandes ciudades ha convertido a las infraestructuras culturales y la política cultural en

3. Pueden consultarse las fuentes en los siguientes enlaces: Observatorio de la Ciudad (https://bit. ly/2yLtE6J); Estadisticas del Ayuntamiento de Madrid (https://bit.ly/2JYnkti); Portal de Transparencia del Ayuntamiento de Madrid (https://bit.ly/2n5rcyf); Madrid Destino (https://bit.ly/ 2tJR19N), y Club de Campo Villa de Madrid (http://transparencia.ccvm.es/).

4. Pueden consultarse las fuentes en los siguientes enlaces: Gaseta Municipal (https://bit.ly/2IwMdLs); Estadistica Ajuntament (https://bit.ly/2tIl3fz); Memòries de l'Institut de Cultura (https://bit.ly/2lEprIQ), y Barcelona dades de cultura (https://bit.ly/2N2dHfq). 
grandes polos de atracción y generadoras de un discurso de marca atractivo en el exterior (Evans, 2003; Rius-Ulldemolins, 2014). Sin embargo, este floreciente interés por la cultura de las élites políticas y económicas no fue acompañado de una política cultural que otorgara mayor autonomía al sector cultural, sino que, al contrario, las ayudas al sector cultural han servido para instrumentalizar, es decir, poner la cultura y los creadores al servicio de los objetivos habitualmente económicos y urbanísticos (Rius Ulldemolins y Zamorano, 2015), lo que ha generado un creciente malestar entre los sectores creativos y los movimientos sociales (Martí-Costa y Pradel i Miquel, 2012; Sánchez Belando y Rius-Ulldemolins, 2014).

Finalmente, la crisis económica y la austeridad han desballestado en gran parte las políticas culturales, especialmente en el sur de Europa, generando un importante debate sobre los objetivos, la legitimidad y los resultados de la acción cultural pública (Rubio Arostegui et al., 2014).

Por todo ello, ha surgido con fuerza, a nivel internacional en general, y en el Estado español en particular, la idea de la necesidad de refundar la política cultural (McGuigan, 2004). Una corriente ha defendido la necesidad de orientar la política cultural hacia la integración social (Belfiore, 2009; Connolly, 2013). Sin embargo, otra corriente especialmente influyente en el Estado español, pero presente a nivel internacional, ha tendido a reconceptualizar la política cultural como una herramienta de antagonismo político antihegemónica que permitiría construir un espacio común, ni privado ni público, en el que los movimientos sociales ganarían el control de las instituciones culturales (Arora, 2015; Gilmore, 2017; Barbieri, 2018). Sin pretender entrar en un debate y conceptualización teórica de esta orientación, este paradigma del procomún — como sí han hecho los autores citados anteriormente- se presenta como un cambio radical, a pesar de retomar gran parte de los postulados de la democracia cultural relativista y contracultural de los setenta (Moulin, 1997). Este además se presenta como un paradigma alternativo a la orientación neoliberal de la política cultural dirigido a la promoción de las industrias cultuales y creativas (Gattinger y St. Pierre, 2010; Murray y Marontate, 2010), pero también de las políticas socialdemócratas orientadas a democratización cultural y la protección social de los creadores organizada por el Estado de bienestar (Menger, 1991; 2005).

Por otra parte, a diferencia de otras corrientes políticas, los movimientos sociales originados a raíz del $15 \mathrm{M}$ y la nueva política en general otorgan, si no un papel central, una importancia significativa a los aspectos culturales y simbólicos, en concordancia con una presencia de intermediarios culturales en el sentido amplio de la palabra. Una visión que les otorga la capacidad de manipular objetos culturales y símbolos, así como los aspectos simbólicos e identitarios en la estrategia política (Levi, 2012; Antentas, 2017). De acuerdo con ello, la visión del rol de la política cultural en este contexto es central como herramienta fundamental en la lucha por la hegemonía. Se trata de una idea que ha permeado en el análisis de la política cultural como una herramienta ideológica y en una visión de que son las ideas y los discursos las que marcan la evolución de las políticas culturales (Barbieri, 2012). No obstante, si bien la política cultural puede considerarse como un discurso utópico que se convierte en práctica reformista en 
la segunda mitad del siglo xx (Dubois, 1999), también es verdad que esta, como otras políticas públicas, son fruto de otros factores y agentes y no solamente de su evolución discursiva, como destacan los analistas del cambio social (Aguilar, 2001).

Sin embargo, esta apuesta por la política cultural procomún se enfrenta a diversos obstáculos conceptuales e institucionales. Por una parte, la política cultural puede interpretarse efectivamente como una arena de lucha política por la hegemonía en términos gramscianos, pero también puede concebirse de modo contrario, como una herramienta de control y normalización por parte del Estado, tal y como se ha hecho desde los estudios culturales (Gray, 2010). Además, por otra parte, desde una perspectiva menos cargada valorativamente y más empíricamente contrastable se puede analizar la política cultural como una acción condicionada por diversos factores y agentes que establecen diversos niveles de definición de objetivos y problemáticas sociopolíticas que son diversas y diferenciadas. Por todo ello, una de las características de la política cultural es su carácter plural y ambiguo en los objetivos - y no dirigido a un solo objetivo contrahegemónico o de dominación-, su débil racionalización en términos weberianos de ajuste de los medios a los fines (normalmente, unos fines muy elevados y unos medios para alcanzarlos notablemente más reducidos), así como su pluralidad y en cierto modo contradicción en sus objetivos al conjugar elitismo artístico con populismo cultural (Moulin, 1997; Dubois, 1999). En este caso, la interpretación de la política cultural no puede presuponer encontrar un solo objetivo como en el paradigma interpretativo anterior, orientado a la dominación o el control, sino un juego plural de conceptualizaciones e intereses, a veces conjugados de forma incoherente y sin conflictos aparentes, y otras veces - la mayoría - representando formas de conflicto larvado o regulado de forma institucional $y$, menos frecuentemente, en pugna abierta y en el espacio mediático.

Por todo ello, en este artículo partimos de cuatro condicionantes que explican la persistencia y la ausencia o la dificultad del cambio social en el campo de las políticas culturales. En primer lugar, uno de los marcos más potentes en los que se ha encuadrado la política cultural desde los ańos noventa ha sido el de la ciudad creativa (Landry y Bianchini, 1995), a partir del cual los gobiernos regionales y locales han basado buena parte de sus estrategias emprendedoras para planificar su desarrollo y su capacidad competitiva a nivel estatal e internacional (Menger, 2010). A pesar de su creciente crítica por su gestión elitista, su carácter instrumentalizador de la cultura y sus efectos socialmente dualizadores y gentrificadores, este paradigma no ha sido abandonado, sino muchas veces escondido bajo retóricas de sostenibilidad y participación cultural (Sánchez et al., 2013).

Por ello, podemos decir que el marco de la ciudad creativa, aun siendo criticado, sigue vigente y no se ha retornado ni mucho menos al marco nacional de difusión de los bienes culturales consagrados, típico de la modernidad avanzadas y del desarrollo de la economía fordista y del desarrollo del Estado del bienestar de posguerra (Urfalino, 1996; Dubois, 2016).

En segundo lugar, la política cultural regional y local no se desarrolla en el vacío institucional, sino que existen unos marcos constitucionales y políticos que 
condicionan las posibilidades de actuación. Asimismo, estos marcos comprometen a las diferentes administraciones entre ellas, al existir financiaciones cruzadas o compromisos institucionales en la gestión de instituciones culturales. Por todo ello, podemos afirmar que la política cultural es un sistema estructurado de relaciones en el que la autonomía completa de una administración para cambiar sus objetivos no existe, al menos a corto plazo (Rius-Ulldemolins y Martinez Illa, 2016; Patricio Mulero y Rius-Ulldemolins, 2017). Por todo ello, el conjunto de agentes públicos (Gobiernos federales o centrales, otros Gobiernos regionales o locales, la UE o los organismos internacionales como la UNESCO) influyen de forma creciente en la política cultural en una relación que va de la cooperación a la competencia abierta (Gray, 2004; RiusUlldemolins et al., 2012; Rosi, 2014; Vos, 2017).

En tercer lugar, existe en la política cultural como en otros sectores de las políticas públicas una intensa tendencia a la inercia administrativa por razones organizativas como el isomorfismo institucional entre las Administraciones públicas y sus agencias, que provoca que las instituciones culturales (museos, auditorios, centros de arte, etc.) se comporten de una forma igual de burocrática que sus titulares, limitando con ello su capacidad de cambio (Gray y Wingfield, 2011; Rius-Ulldemolins, 2014a). Asimismo, esta inercia viene reforzada también por la tradicional independencia de las instituciones culturales, que han basado en el capital simbólico de sus directores artísticos una herramienta para resistirse a los intentos de intervención de los responsables políticos y para enfocar estas instituciones a los objetivos de prestigio artístico y para resistirse a los intentos de alinear estas agencias públicas con intereses de valor público (Rius-Ulldemolins, 2013). En este sentido, los cambios en los responsables políticos y los programas de reforma de estas tienen escasa influencia, al menos a corto plazo (Rius-Ulldemolins y Rubio, 2013). Asimismo, en la política cultural existen - aunque pequeños económicamente- influentes lobbys del sector cultural que condicionan la política cultural y, por lo tanto, constituyen una inercia alrededor de los intereses creados respecto a las ayudas públicas (Rubio Arostegui, 2014; Rius-Ulldemolins y Martinez Illa, 2016).

Y, por último, la política cultural ha ganado centralidad en las últimas décadas en relación a su importancia como sector económico y por su papel clave en las estrategias locales de regeneración y branding urbano y el turismo, lo que la ha resituado en un lugar central en la agenda política. Ello ha comportado efectos beneficiosos para atraer una mayor atención de la agenda pública, pero ha conllevado que sus actuaciones se hayan convertido en foco de atención política y mediática (Rius Ulldemolins y Zamorano, 2015; Rubio y Rius-Ulldemolins, 2015). Asimismo, con ello los partidos políticos han situado en la lógica competitiva electoral a los sectores culturales afines como objetivo de protección y de recursos simbólicos para su construcción discursiva y a los no afines como sectores objeto de críticas y de castigos en términos presupuestarios, reproduciendo así la lógica clientelar en diversos sectores políticos (Rius-Ulldemolins y Martinez Illa, 2016; Rius-Ulldemolins et al., 2017).

En definitiva, en este artículo nos proponemos demostrar la fuerza de la herencia recibida y de la inercia histórica como factor interpretativo de las políticas culturales, 
una hipótesis ya avanzada por Menger (2011): «En los países que han desarrollado vigorosamente su política cultural después de medio siglo, la intervención se ha desarrollado en gran medida por adición y sedimentación, y muy poco por sustitución radical de objetivos y de prioridad de presupuestarias, por lo que concierne a los grandes pilares de la intervención». Esta inercia no solo se explica por factores exógenos —la estabilidad institucional — o endógenos como la inercia del marco explicativo, sino también, como indica Dubois (2016), por la pervivencia de la legitimidad del capital simbólico del campo artístico que fomenta las políticas de la democracia cultural como difusión de la cultura. Una política que, aunque puesta en duda por los recientes cambios en el ámbito digital, continúa en buena parte basada en las políticas clásicas de difusión cultural dirigida a las capas medias de la población (Ariño Villarroya, 2010).

Por otra parte, esta política pública dista de ser coherente y racionalmente orientada a causa del carácter difuso de su objeto de promoción, lo que comporta numerosas limitaciones y barreras organizativas al cambio (Gray, 2009). Asimismo, podemos ver cómo a nivel periodístico, o por parte de ciertos sectores, se exagera la magnitud del cambio, por ejemplo respecto a la reorientación neoliberal de las políticas culturales de las últimas décadas (Gattinger y St. Pierre, 2010; Murray y Marontate, 2010; Hesmondhalgh et al., 2015). Por todo ello, entendemos que la relación entre cambio político y política cultural no ha sido suficientemente estudiada al centrarse mayoritariamente en el cambio y no la persistencia. Sin embargo, como observa Nisbet, en la historia humana - y ello puede aplicarse a la política cultural también- es más común la persistencia que el cambio (Nisbet y Kuhn, 1993). Por ello, a continuación mostraremos cómo en los casos de Madrid y Barcelona los cambios discursivos en la política cultural en las grandes ciudades no han ido acompañados de un cambio equivalente en sus dimensiones organizativas y sistémicas.

\section{CAMBIO POLÍTICO, SISTEMA CULTURAL Y CAMBIO EN LAS POLÍTICAS CULTURALES: EL PESO DE LOS MODELOS HEREDADOS}

\section{Madrid: el peso de la capitalidad y las batallas culturales}

a. Situación de partida: contexto político-administrativo y sistema cultural

Madrid constituye hoy en día una capital cultural debido a su concentración de patrimonio cultural, sectores y actividad cultural profesional. Su dinámica viene muy condicionada por tres elementos: a) su condición de capital de un Estado que, a pesar de ser descentralizado, concentra las instancias de poder político y económico; b) la creciente concentración de la industria cultural y creativa, y c) también una creciente concentración de sectores y profesionales creativos.

En primer lugar, por lo tanto, su sistema local de política cultural viene muy caracterizado por su proximidad con los poderes del Estado. Así, la inmensa mayoría de las 
grandes instituciones culturales de la Administración General del Estado, al que esta administración le dedicaba en 2013 unos 114 millones de euros, concentraba la gran mayoría de las recursos aportados a las instituciones culturales españolas y un $23 \%$ del total de su gasto cultural ministerial (Rius-Uldemollins y Zamorano, 2014). Esta presencia del Estado, que podemos entender como continuadora del mecenazgo real de la Ilustración tardía, ha facilitado en gran manera que la política cultural desarrollada en la capital española haya sido del tipo centroeuropeo definido por Zimmer y Toepler (1996), es decir, con una gran presencia y legitimidad de la alta cultura, muy centralizada y dirigista y poco impermeable a la influencia de la sociedad civil.

De manera paralela, podemos afirmar que la política cultural local no ha sido en Madrid una prioridad. Como analiza Rubio Arostegui $(2003$; 2005) el desarrollo de la política cultural en Madrid desde los años ochenta se caracteriza por tres elementos: a) un fenómeno de path dependence en el que la gestión cultural autonómica y local está muy condicionada por la presencia de las estructuras del Ministerio de Cultura en la ciudad; b) una gran influencia de los sectores artísticos profesionales (especialmente las artes escénicas) en las decisiones y prioridades culturales, y c) un alto grado de consenso en torno a los objetivos, al no existir un debate identitario como en otras comunidades autónomas y articularse un cierto consenso sobre las grandes líneas de desarrollo entre los dos grandes partidos de gobierno (PSOE y PP) en los tres niveles de Administraciones públicas en Madrid, local, autonómico y estatal (Rubio Arostegui, 2012). Una unanimidad que en la actualidad ha sido caracterizado como la «cultura de la transición» (Martínez, 2012), un paradigma de política cultural que enfatiza el consenso en torno al régimen del 78 y que habría marginado a la cultura crítica con sus olvidos históricos, compromisos políticos y renuncias sociales. Fue a través de este acuerdo como Madrid se potenció como capital cultural desde los ańos ochenta, a partir de la conocida movida madrileña, una dinamización cultural de carácter apolítico, y se consolidó en los años noventa con la liberalización, desarrollo y concentración de las industrias culturales y mediáticas en la capital del Estado.

Este proceso desbancará a Barcelona, que había sido la punta de lanza cultural y lugar de introducción de las vanguardias en el contexto cultural español (Bouzada, 2007). Esta transformación se vio reflejada, por ejemplo, con la capitalidad artística conseguida a partir de la feria cultural ARCO impulsada por IFEMA, el Ayuntamiento y la Comunidad de Madrid, que impulsó y simbolizó la modernización y dinamización de la escena artística madrileńa a partir de los años ochenta (Ramoneda, 1997). Fueron, sin duda, factores de carácter económico y político los más influyentes en este cambio. Entre ellos destacan la concentración en Madrid de las sedes de las corporaciones multinacionales y financieras que tradicionalmente han desarrollado una labor de mecenazgo en las artes visuales; la presencia de las grandes instituciones culturales del Ministerio de Cultura con importantes presupuestos para la adquisición de obras, que compran en las galerías de arte de esa ciudad (UAGAE, 2002), etc. Si a este liderazgo simbólico en la innovación artística, le unimos la aglomeración en Madrid de un $30 \%$ de los efectivos de profesiones creativas (especialmente de aquellos relacionados con las industrias culturales, como la industria audiovisual con un $34 \%$ y la 
industria editorial con un 38,47 \%) y de la práctica totalidad de las sedes de los grupos mediáticos privados, podemos comprender la importancia del sector cultural en la ciudad de Madrid (Méndez et al., 2012).

b. Orientación de la política cultural local desde el año dos mil

Uno de los desarrollos recientes destacables de la política cultural local de la capital del Estado es el Plan Estratégico de Cultura de Madrid 2012-2015 (PECAM), que significaría, al menos sobre el papel, una evolución del modelo de planificación cultural en las grandes ciudades. A diferencia de sus planes estratégicos culturales en Barcelona (1999 y 2006), sus antecedentes en planificación en grandes ciudades de España, adoptó una orientación inclusiva de diversos actores sociales, al menos en lo discursivo. Así pues, a diferencia de los planes estratégicos de Barcelona, más orientados a la participación sectorial, el PECAM se manifestaba favorable a experiencias de «abajo hacia arriba» como las de La Tabacalera de Lavapiés, el Campo de Cebada, Medialab Prado o Esta es una Plaza (PECAM, 2012-2015).

Si en el caso barcelonés el Plan Estratégico de 1999 surgía de una comprensión de la cultura como recurso para las políticas de desarrollo urbano y en una nueva etapa de la política cultural caracterizada por alianzas pública-privadas en la movilización de la cultura (ICUB, 1999), el Plan Estratégico de Cultura de Madrid se dirigió más a una potenciación de la capacidad de generar valor cultural a partir de otorgar espacios de libertad a los creadores en los que pudieron desarrollar un coworking multidisciplinar e innovador (Ayuntamiento de Madrid, 2012). Sin embargo, aunque la mayoría de estos espacios eran autogestionados por colectivos vinculados al procomún, no contaban con un acuerdo a medio o largo plazo por parte de las Administraciones públicas. Ello conllevó que espacios como Medialab Prado, con una reputación importante dentro del campo de los espacios de innovación cultural y social, vieran amenazada su continuidad. Así, en marzo de 2014, el Ayuntamiento de Madrid del PP tomó la decisión de desahuciar al colectivo que lo gestionaba y cederlo a la empresa Telefónica para poder desarrollar su proyecto Open Future, consistente en la creación de un clúster creativo y tecnológico, mostrando así su debilidad frente a instrumentalizaciones partidistas y corporativas de estas iniciativas. Esta decisión del Gobierno local, liderado por Ana Botella, generó una oleada de críticas que consideraban la transmisión de poderes como una privatización opaca de un espacio público. Con esta argumentación, los colectivos culturales y los movimientos sociales consiguieron paralizar el proyecto. Podemos ver por lo tanto cómo en el marco del Gobierno del PP se desarrollaron de forma contradictoria algunas experiencias participativas, aunque siempre en una situación dependiente de las decisiones top-down de los responsables políticos.

Sin embargo, el giro neoliberal en la política cultural local durante el mandato popular parece claro en la forma de implementar la política cultural y sus prioridades de gasto. Desde el año 2013, el Área de las Artes incorporó las competencias de Deportes y Turismo. Ello en sí no constituye un tipo de agregación inusual en política cultural, pero sí lo es el hecho que gran parte de los fondos se destinaban a la empresa 
Madrid Destino Cultura Turismo y Negocios. Esta empresa, surgida de la fusión de Madrid Arte y Cultura S. A. (MACSA) absorbió a Madrid Visitors and Convention Bureau S. A. (empresa encargada de la promoción del turismo y los congresos empresariales y profesionales), y cuenta con un presupuesto de 54 millones de euros, es decir, el $51 \%$ del gasto del área que alcanza en 2014 los 105 millones (y el $96 \%$ del capítulo IV de transferencias), de los que una parte sustancial están destinados a promoción económica y no a actividades propiamente culturales (Ayuntamiento de Madrid, 2013). Asimismo, su fusión con la empresa Madrid Espacios y Congresos S. A., que gestiona espacios polivalentes, representó una mayor disolución de los objetivos de la política cultural.

c. El cambio político en 2015 y obstáculos exógenos al cambio de las políticas culturales locales

Esta dinámica de instrumentalización económica de la cultura parecía que iba a cambiar después de la victoria de Ahora Madrid en los comicios locales del 2015 y el surgimiento de un Gobierno de coalición de izquierdas con el PSOE. Ya en la toma de posesión de algunos concejales de Ahora Madrid se explicitaba este afán de cambio, mediante la expresión utilizada en el siglo Xvi por la revuelta campesina liderada por Thomas Müntzer omnia sunt comunia. Entre estos se encontraba Guillermo Zapata, el edil elegido por Manuela Carmena, alcaldesa de Madrid, para gestionar la cultura de la capital del Estado. Zapata era un activista y creador del espacio autogestionado El Patio Maravillas, uno de los espacios comunes más significativos de Madrid y que cerró sus puertas en julio de 2017, una década después de su nacimiento. No obstante, su mandato tan solo duró dos días, ya que Carmena lo destituyó tras hacerse públicos unos tuits de humor negro lanzados por el concejal años antes, convirtiéndose, a la vez, en el primer desacuerdo importante en el seno del recién inaugurado «ayuntamiento del cambio».

Así pues, este fue el primer titular protagonizado por la gestión del Área de Cultura del nuevo Gobierno local madrileño. El discurso de la cultura de lo común, la democratización cultural, la descentralización, que encontramos en diferentes documentos de Ahora Madrid, como en su programa electoral o el mapa estratégico de sus políticas culturales, ha sido empañado en primer lugar por los desacuerdos internos, las decisiones políticas controvertidas y la continuidad de ciertas dinámicas de gestión administrativa, y en segundo lugar no ha ido acompañado de una financiación cultural adecuada en los presupuestos municipales.

Asimismo, la gestión cultural de la capital ha concentrado buena parte de los ataques de la prensa de la capital, en buena medida hostil al nuevo Gobierno, generando lo que se ha llamado "guerras culturales». De este modo, la concejala de Cultura Celia Mayer ha sido objeto de numerosas polémicas. En las navidades de 2015, fueron muy criticados los trajes que los Reyes Magos exhibieron en la cabalgata de la ciudad y meses después saltó a la opinión pública la llamada "crisis de los titiriteros», la cual llevó a prisión a los miembros de una compañía de teatro del guiñol por 
enaltecimiento del terrorismo, cuya causa fue finalmente archivada. La aplicación de la Ley de Memoria Histórica por Mayer tampoco estuvo exenta de polémica. La concejala ordenó retirar monumentos franquistas de la capital de manera unilateral, sin que el Ayuntamiento hubiese desarrollado una normativa municipal que indicase cómo aplicar esta legislación. Para evitar convertir estas actuaciones en una enésima «batalla cultural» la alcaldesa retiró estas competencias del Área de Cultura. Pero, la gota que colmó el vaso fue la decisión de los directivos de Madrid Destino, bajo la aprobación de la edil, de quitar los nombres de los dramaturgos Max Aub y Fernando Arrabal a dos pabellones dedicados al teatro de texto del centro de arte municipal Matadero. Esta decisión vino motivada por el cambio de temática de las salas donde la nueva dirección del Matadero quería impulsar una programación centrada en las "artes vivas»; danza urbana, performances y disciplinas experimentales. Las críticas generadas por esta decisión, sobre todo en el sector teatral —uno de los sectores con más poder en las dinámicas culturales de la capital— derivó finalmente en la destitución de Celia Mayer como responsable del Área de Cultura, asumiendo esta responsabilidad la propia alcaldesa.

d. El cambio en la política cultural local a partir de 2015: alcance de la trasformación y limitaciones endógenas al cambio

En términos generales presupuestarios se puede observar una lenta mejoría en los presupuestos culturales. En el último periodo del Gobierno local del PP (2011-2014) estos no superaron los 130 millones de euros, un 2,6\% respecto del total de presupuestos del Ayuntamiento de Madrid. Por el contrario, a partir del 2015 se observa una lenta pero continuada mejoría en términos absolutos, hasta alcanzar los 159,3 millones de euros en 2017 y un porcentaje de media en el periodo de 2015 a 2017 de un 3,3\%, es decir, 0,7 décimas más de esfuerzo cultural en el gasto local público en la capital del Estado (Ayuntamiento de Madrid, 2018). Sin embargo, si observamos no tanto el cuánto sino el cómo se implementa el gasto cultural, observamos una significativa continuidad.

En primer lugar, uno de los focos de la política cultural local ha sido la empresa Madrid Destino, que había recibido no pocas críticas por parte de los miembros de la candidatura de los comunes por considerarla una gran organización vertical y orientación neoliberal (Rowan, 2015). Esta empresa municipal gestionó 53,3 millones de euros el ańo 2016, es decir, el 31,7\% del presupuesto total de cultura del Ayuntamiento de Madrid. Desde su nacimiento en el ańo 2014, Madrid Destino es una pieza clave de la gestión cultural de la ciudad y, en consecuencia, no solo sus partidas dentro de los presupuestos han ido incrementándose, sino que el nuevo Ayuntamiento le ha encomendado la organización de nuevas actividades culturales en la ciudad $^{5}$. Sin embargo, pese a la subida presupuestaria comentada, el porcentaje que

5. Cuentas anuales Madrid Destino, 2015 (https://bit.ly/2KqdEIB). 
gestiona Madrid Destino del total de presupuesto de cultura no ha variado en gran medida desde que se creó: $31,7 \%$ en 2014, 29,9 \% en 2015, 36,07 \% en 2016, mientras que para 2017 se prevé un 31,7 \% (Ayuntamiento de Madrid, 2018). Celia Mayer, consciente de la centralidad de la empresa, se puso como objetivo su reorganización orgánica y directiva para que esta se reorientase hacia la cultura promulgada por Ahora Madrid, es decir, desde una perspectiva del «bien común». Sin embargo, durante los dos años que Mayer lideró el Área de Cultura, lo único que consiguió fueron críticas y presiones desde la oposición política del consistorio, lo que dio como resultado despidos, destituciones y abandonos desde el organigrama directivo y gestor de Madrid Destino.

Ciertamente, se ha intentado transformar la organización y se ha aplicado una de las propuestas de "potenciar la iniciativa ciudadana en el uso de espacios e infraestructuras, la programación de contenidos y la organización de eventos en las instalaciones municipales ${ }^{6} »$. Con ese propósito nace Imagina $\mathrm{Madrid}^{7}$, un programa impulsado por Intermediae (otra de los espacios gestionados por Madrid Destino). Este proyecto pone en marcha procesos de creación colectiva entre la ciudadanía y el tejido artístico, para transformar nueve lugares públicos de la ciudad mediante la intervención urbana y la activación cultural sin olvidar su historia e identidad, convirtiendo el espacio público en un laboratorio. Además, los espacios elegidos para su transformación están sitos en distritos periféricos, que pretenden descentralizar la cultura construyendo una ciudad policéntrica.

Siguiendo con la descentralización de la cultura como uno de los principios rectores de las políticas culturales del Gobierno de la capital, desde el Área de Cultura se adoptó, con la intención de rediseñarlo y ampliar su alcance, un programa del anterior Gobierno del PP: ¡Madrid Activa! Entre los objetivos de este programa está el de "potenciar la cultura de proximidad apoyando nuevas propuestas y nuevos espacios para la cultura en la búsqueda del equilibrio territorial desarrollando una actividad cultural dinámica y plural que atienda a la mayor diversidad de intereses». Para tal fin el Ayuntamiento de Madrid presupuestó 3,4 millones para el ejercicio 2017 y en el anuncio de la convocatoria para la presentación de las propuestas culturales por parte de las personas creadoras y productoras, se explicaba la ampliación de las áreas de creación con respecto a anteriores convocatorias. No obstante, tanto en la edición del 2016 como en la actual, los procesos de evaluación y publicación de resultados del concurso público generado por ¡Madrid Activa! han sido largos y tediosos, lo que ha suscitado nuevamente críticas tanto de los sectores y personas participantes como de las empresas. El Área de Cultura aludió a problemas jurídicos-administrativos junto a normativos para justificar la demora de resultados, ya que la legislación para contratación de personal heredada por el PP dificulta y, en ocasiones, obstaculiza el fluir del Área y los proyectos propuestos. $\mathrm{Al}$ mismo tiempo, otras iniciativas muestran un

6. Programa electoral Ahora Madrid, pto. 3.4.2. c.

7. https://www.imagina-madrid.es/es. 
contraste entre discurso y actuaciones: este es el caso de la creación de equipamientos como el programa DAM, orientado a la excelencia pedagógica artística al alcance de la ciudadanía $^{8}$, y que representa un caso de cómo el discurso de innovación y excelencia desarrollado - siendo la excelencia pedagógica algo esencialmente elitista, destinado para maestros o profesionales de las artes escénicas - entra en conflicto con un objetivo democratizador para todos los públicos.

Ciertamente, el esfuerzo democratizador en lo cultural y la potenciación de la cultura de proximidad se han visto reflejados en su inversión en bibliotecas y el gasto cultural en los distritos. Actualmente, la red de bibliotecas públicas de Madrid cuenta 32 equipamientos de titularidad municipal y 16 de titularidad autonómica. El Ayuntamiento de Madrid ha presupuestado en 2017 4,2 millones para ocho nuevas bibliotecas y entre sus objetivos está renovar y ampliar las exposiciones y actividades en estos equipamientos culturales. Así, el Gobierno de Madrid tiene previsto gastar 8,37 euros por habitante en bibliotecas públicas y patrimonio bibliográfico, incrementando en una media de 1 euro más la cantidad en relación con los cinco años anteriores. No obstante, el gasto cultural por habitante sigue siendo muy inferior respecto a otras ciudades como Barcelona (Ministerio de Educación y Cultura, 2016).

En cuanto a las actividades culturales, una parte importante de las cuales son gestionadas desde los propios distritos, han descendido considerablemente en los últimos cinco años. Así, observamos que en el ejercicio 2011 fueron destinados 78,5 millones de euros a actividades culturales, de los cuales 33109793 eran gestionados por los distritos, es decir, un 44,53 \% del total. A partir de este año empieza un descenso que llega a su máxima bajada en el 2014 con un presupuesto de 29,5 millones de euros, de los cuales el 81,28 \% (23 518110 euros) es gestionado desde los distritos y el 19,14 \% (6 767110 euros) por el Ayuntamiento. Durante los siguientes ejercicios (20152017) hay una mejoría, hasta llegar a los 50,7 millones de euros presupuestados para el actual 2017, de los cuales está previsto que los propios distritos gestionen 35906921 $(70,77 \%)$. En definitiva, el gasto de los distritos aumenta en cifras generales, recuperando el gasto anterior a 2011, pero no en términos porcentuales, con lo que no parece reflejar la prioridad del gasto en cultura de proximidad.

Finalmente, desde la llegada al Gobierno de Carmena y su equipo, las cantidades presupuestadas para el Área de Cultura se han incrementado paulatinamente con respecto al Gobierno liderado por Ana Botella. En 2016 las cantidades destinadas a cultura aumentaron un $12,6 \%$ respecto al 2015 , pasando de 108,5 millones de euros a 122,2 . Sin embargo, pese a que esta cantidad es la más alta en los últimos cinco años, se queda muy por debajo de los 161,6 millones de euros que se gastaron en 2011. No obstante, la cifra del gasto público en cultura no nos muestra adecuadamente la importancia de las políticas culturales para un Gobierno si no evaluamos el porcentaje que ocupa en el global del presupuesto. Así vemos que, pese al discurso empleado por la

8. DAM. Centro de Innovación Pedagógica en Danza, Artes Escénicas y Música (https://bit. ly/2Kw2JNB). 
nueva política en el Ayuntamiento madrileño, las cantidades destinadas a cultura en 2016 apenas llega al $3.98 \%$ sobre el total presupuestario.

En definitiva, el análisis de las cifras y los discursos desarrollados sobre la política cultural nos muestran una clara intención inicial de realizar cambios importantes que, finalmente, por diferentes causas exógenas y endógenas no han llegado a materializarse, creando frustraciones y más conflictos, no solo dentro del propio consistorio, sino también entre los sectores y la opinión pública. Desde el consistorio se avanza hacía nuevas políticas culturales, pero encuentran muchos obstáculos administrativos, económicos y también ideológicos. En el programa de Ahora Madrid encontramos dos propuestas que abogaban por «crear nuevos protocolos y procedimientos administrativos (licencias, reglamentos, trámites, concursos, convocatorias) para una gestión de la cultura más ágil, flexible, transparente», pero también por «crear una Concejalía de Cultura independiente [...] dotada de presupuesto suficiente, adaptando sus órganos de gobierno a una gestión transparente y democrática» ninguna de estas propuestas han sido puestas en marcha y tal vez serían facilitadoras de nueva políticas culturales.

\section{Barcelona: el modelo como constreñimiento}

a. Situación de partida: contexto político-administrativo y sistema cultural

La ciudad de Barcelona es internacionalmente conocida por haber desarrollado un modelo de ciudad liderado por el Gobierno local, con el objetivo de transformarla económica, social y culturalmente después del franquismo (González, 2011; Rius-Ulldemolins y Sánchez, 2015). Asimismo, se ha analizado el impulso modernizador de la ciudad bajo los sucesivos Gobiernos socialistas, inicialmente de orientación socialdemócrata en los ochenta y posteriormente con una orientación más socioliberal en los noventa y dos mil (Marshall, 2000). El Gobierno local se caracterizó en sus primeras etapas por la aplicación de un urbanismo socialmente inclusivo en los barrios periféricos de un centro históricamente degradado (Rius-Ulldemolins, 2014), con una utilización destacada de la cultura para la regeneración urbana (Subirós, 1999, J. Rius-Ulldemolins, 2006).

Para algunos, el modelo Barcelona es un modelo de éxito en el que se combinaron de forma acertada renovación urbana y planificación cultural (Subirós, 1999). Sin embargo, desde su formulación como modelo, diversos autores han desarrollado diferentes críticas por la deriva mercantilista, elitista y autoritaria del modelo (Degen y García, 2012).

Políticamente, la ciudad de Barcelona ha estado dominada por el partido socialista tras la dictadura franquista: las primeras elecciones municipales democráticas de 1979 dieron la victoria al Partido de los Socialistas de Catalunya (PSC), pero tuvo que gobernar en alianza con los comunistas (PSUC). Sin embargo, a partir de los años ochenta los socialistas gobernaron la ciudad con cómodas mayorías y de forma ininterrumpida hasta 2011. En este ańo, se interrumpe esta etapa con la victoria de 
Convergencia i Unió, una coalición de la derecha conservadora y catalanista. Sin embargo, esta etapa dura poco tiempo y en las elecciones de 2015 el partido liderado por Ada Colau, Barcelona en Comú (BEC), fue el primer partido en las elecciones, y de 2016 a 2017 ha gobernado en coalición con el PSC. A finales de 2017 rompe este pacto y vuelve a gobernar en solitario.

\section{b. Orientación de la política cultural local desde el año dos mil}

El modelo Barcelona de políticas culturales se caracteriza por: a) el liderazgo de la Administración local muy por encima de la definición competencial en cultura y que se evidencia a nivel organizativo, presupuestario y en la gobernanza de las instituciones culturales; b) la utilización de la regeneración urbana como oportunidad de desarrollo de una mayor gobernanza cultural; c) la utilización de grandes eventos y años temáticos para ganar centralidad social para el sector cultural; d) creciente importancia de la cultura en la planificación estratégica local y la elaboración de planes estratégicos culturales como factor de potenciación de la cooperación público-privada y del desarrollo de los sectores culturales en su conjunto, y e) la institucionalización de la participación ciudadana en cultura con la cogestión de equipamientos de proximidad o el establecimientos de mecanismos consultivos como los consejos de las artes, inspirados en el modelo anglosajón de arm's lenght (Rius-Ulldemolins y Rubio, 2013). Por ello, analizaremos ahora diferentes casos que nos servirán como casos significativos de análisis de las diferentes dimensiones del modelo Barcelona de política cultural: la agencialización y centralidad de la política cultural, las organizaciones culturales nacionales, los planes estratégicos, la regeneración urbana y la creación del clúster cultural del barrio del Raval, el Fórum de las Culturas 2004, los centros culturales de proximidad y el Consejo de la Cultura (Sánchez Belando y Rius-Ulldemolins, 2014, J. Rius-Ulldemolins y Sánchez, 2015).

c. El cambio político en 2015 y obstáculos exógenos al cambio de las políticas culturales locales

La entrada en el nuevo Gobierno en 2015 fue tomada con inquietud, especialmente por aquellos sectores relacionados con las grandes instituciones e industrias culturales de la ciudad, que temían recortes significativos de financiación, en concordancia con las críticas desarrolladas por el núcleo de cultura del nuevo partido de Barcelona en Comú, que los tachaba de elitistas y neoliberales y apostaba por una cultura en común, concretada en la autogestión de los centros culturales y centros cívicos, en la mejora de las condiciones laborales en los equipamientos culturales para los creadores y en la transformación del Consejo de la Cultura Local en un órgano decisorio e gestor de políticas y no consultivo como es actualmente (Barcelona en Comú, 2015).

Dentro de esta campaña crítica se alinearon sectores de la élite ciudadana y sectores mediáticos locales importantes (Barranco et al., 2015), lo que generó una inquietud y parálisis en el nuevo Gobierno, necesitado de apoyos por su situación minoritaria en 
el pleno municipal. Para responder a esta situación de incertidumbre, que duró más de tres meses sin nuevo responsable cultural, se acabó nombrando como comisionada (pero no regidora, es decir, sin ser miembro del Gobierno) a Berta Sureda'. Esta no era en absoluto desconocida en el mundo de la política cultural al haber ostentado diversas responsabilidades — como gerente del $\mathrm{CCCB}$ - durante el mandato socialista. Este nombramiento fue considerado como una primera renuncia a un cambio radical en la política cultural y, en este contexto enrarecido, Sureda empezó un tímido programa de cambios en la gestión cultural. Sin embargo, antes de fructificar y a solo un año de haber iniciado el proyecto, fue destituida en julio de 2016 y acabó con ello el mandato de los comunes en cultura.

La razón principal de esta destitución fue en gran parte la necesidad de alcanzar pactos de gobierno con otras fuerzas, en este caso el PSC. Sin embargo, este cambio refleja asimismo la dificultad de pasar de la teoría a la práctica en el marco de una debilidad política y la inercia del modelo cultural y la importancia concedida a la cultura por parte de otros sectores políticos como los socialistas como parte integrante de su modelo de ciudad. Todo ello se vio reflejado en que el nuevo teniente de alcalde y jefe de filas socialistas quisiera asumir personalmente la responsabilidad de cultura, promoviéndola otra vez al rango de regidoría — perdida durante el breve mandato de los comunes- e incluyéndola en su cartera de Empresa e Innovación. Inmediatamente expuso 34 medidas de choque para la cultura. Finalmente, Collboni decretó que el nuevo gerente del ICUB y, por lo tanto, jefe ejecutivo en el Área Cultural, sería Xavier Marcé, antiguo responsable del Instituto de Industrias Culturales autonómico durante el mandato socialista y, posteriormente, alto directivo de una de las empresas más importantes en las industrias culturales de la ciudad y presidente de la patronal de las artes escénicas catalana ADETCA ${ }^{10}$.

Sin embargo, «los tiempos habían cambiado» y este nombramiento generó un elevado malestar en Barcelona en Comú y los sectores culturales que habían apoyado la candidatura, que denunciaron el carácter a favor de las empresas de este responsable y sus conflictos de intereses al representar un caso de puerta giratoria en la gestión pública. Este cambio de la cartera de Gobierno generó una protesta por parte de las asociaciones de profesionales de la cultura por lo que juzgaban una injerencia de las empresas privadas, la falta de control y transparencia de los concursos de los festivales

9. Berta Sureda, miembro de la lista electoral de Ada Colau en 2015, dista de ser una outsider de la política cultural y su trayectoria va ligada durante los últimos veinte años a la gestión pública de la cultura en diversas responsabilidades ligadas al entorno socialista: empezó como coordinadora de exposiciones del Instituto de Promoción Urbanística (1988) y la Olimpiada Cultural (1989-1992) y, también, de actividades escénicas del Festival de Otońo y el Festival de las Artes de Barcelona. También trabajó como responsable de actividades culturales del Centro de Cultura Contemporánea de Barcelona durante el mandato de Josep Ramoneda, y durante el mandato de la socialista Caterina Mieras estuvo al frente de la Entidad Autónoma de Difusión Cultural del Departamento de Cultura de la Generalitat de Catalunya.

10. Associació d'Empreses de Teatre de Catalunya (ADETCA). 
e instituciones culturales de la ciudad y la precariedad de los trabajadores independientes de la cultura. El manifiesto, que recibió 1400 adhesiones, reflejaba la frustración por el abandono del Gobierno de Colau de todo intento de transformación de fondo del modelo de política cultural y lo que ellos interpretaban como una traición de los comunes respecto de las promesas respecto de una política cultural más favorable al sector «neobohemio» (Treballadors/es independents de la cultura, 2016). Frente a estas protestas, el PSC nominalmente paralizó el nombramiento de Marcè como director del ICUB, pero fue nombrado meses después como asesor especial del alcalde para dirigir políticamente el área y asistir a las reuniones en nombre del Ayuntamiento (ICUB, 2017). Es decir, de forma discreta los sectores culturales con mayor peso económico y político retomaron el control de la agenda pública de la cultura.

d. El cambio en la política cultural local a partir de 2015: alcance de la trasformación y limitaciones endógenas al cambio

Una característica del modelo Barcelona de política cultural es su alto grado de agencialización, desarrollado para garantizar su independencia de los ciclos políticos, dotarla de flexibilidad en la gestión y aproximarla al sector privado lucrativo y no lucrativo. Después de su creación, el Instituto de Cultura de Barcelona (ICUB) pasará de gestionar el $14 \%$ en 1990 al $85 \%$ el 2000, porcentaje que se ha mantenido estable con leves oscilaciones hasta 2016, debido al nivel de inversión que gestiona directamente el Ayuntamiento (Ajuntament de Barcelona, 1980-2017). En cuanto a la centralidad de la cultura en la agenda política local, tras una etapa de descenso desde 2010, podemos observar un ligero aumento en el periodo de 2014-2017, en el que se estabiliza con un 5,3\% de media. Por tanto, las variaciones en la importancia concedida a la cultura y el porcentaje de gasto cultural no parecen ir ligado a consideraciones ideológicas de los Gobiernos, sino en gran parte a la coyuntura económica y presupuestaria del Gobierno local.

Esta estabilidad viene confirmada por el volumen y las prioridades del gasto cultural. En términos generales, podemos observar un leve aumento del gasto cultural, que en el periodo convergente entre 2011-2014 fue de un 4,9\% del global del presupuesto del Ayuntamiento de Barcelona. A partir de 2015 el presupuesto aumenta desde los 136,6 millones de euros hasta los 147,8 en 2017. Sin embargo, el aumento del porcentaje en relación con el presupuesto global en el periodo 2015-2017 es de tan solo 0,4 décimas, un 5,3\% del total. Por una parte, podemos ver la primacía en el gasto en los grandes equipamientos culturales bajo la forma de consorcios y fundaciones orientados a la promoción de la alta cultura. En estos se gastó en 2001 23,3 millones de euros (25,8\%), mientras que en 2010 la cifra aumentó hasta los 32,3 millones (28,2\% del total). Esta cifra siguió ascendiendo hasta los 35,2 millones en 2014 (26,6 \% del total) en la etapa de la derecha, pero tampoco bajó ya en la etapa de Barcelona en Comú, hasta alcanzar la cifra récord de 35,2 millones $(28,2 \%$ del total) en 2016. Con ello se evidencia que la asociación entre Gobiernos de la derecha, con un menor gasto cultural, o bien Gobiernos de nueva izquierda, con un gasto 
cultural menos orientado a las instituciones de la élite cultural, no ejecutan grandes cambios en la práctica presupuestaria. Por el contrario, observamos una continua estabilidad e inercia y, si acaso, una tendencia incrementalista a largo plazo. En el otro espectro de la política cultural, en el ámbito de la participación y de la cultura de proximidad más cercana al paradigma de la democracia cultural defendida por el izquierdismo antiautoritario (Urfalino, 1996), los cambios tampoco son muy sustantivos: desde 2010 el gasto efectuado en el programa de Centros Cívicos supera los 11,5 millones de euros y llega a su máximo en 2017 con un gasto de 15,2 millones. $\mathrm{Si}$ analizamos la media del gasto en cultura de proximidad en relación con el periodo de gobierno podemos observar cómo se observa una cierta progresión: 8,6 \% en el último periodo del PSC (2010-2011); 9,7\% en el periodo de CiU (2012-2015), y en el de BEC sube tres décimas hasta llegar al 10\%. Podemos observar, por lo tanto, una leva variación incrementalista, especialmente en el gobierno de BEC, pero que se detecta tanto en Gobiernos de derecha como de izquierda. En este caso, por lo tanto, nos encontramos con un programa que genera consenso entre los diferentes equipos de gobierno de la cultura de la ciudad.

Por otra parte, los grandes equipamientos culturales muestran cómo en el modelo que se ha configurado de política cultural, el Gobierno local ha ejercido un rol de liderazgo y cómo se ha establecido un sistema de gobernanza multinivel junto con el Departamento de Cultura y el Ministerio de Cultura (Rius-Ulldemolins, 2014b). Muestra de ello es que el Ayuntamiento de Barcelona, sin tener competencias específicas que le obliguen a ello, aporta casi la mitad que el Gobierno autonómico, unos $30,2$ millones (un $22 \%)^{11}$. Además, el Ayuntamiento de Barcelona está presente en las estructuras de gobernanza de diez de los doce equipamientos nacionales (Auditori, Centre de Cultura Contemporànea de Barcelona, Fundació Antoni Tàpies, Fundació Miró, Liceu, Museu d'Art Contemporani de Barcelona, Mercat de les Flors, MNAC, Palau de la Música, Teatre Lliure, Teatre Nacional de Catalunya). Esta configuración sitúa al Ayuntamiento de Barcelona con un rol de gran centralidad, pero también le resta en gran medida capacidad de control y discrecionalidad en la gestión cultural (Rius-Ulldemolins, 2014b).

Las formas de la gestión cultural revelan muchas veces de forma más fidedigna las orientaciones y las prioridades de la política cultural que los discursos. En concordancia, el análisis del organigrama de la política cultural barcelonesa muestra una elevada coherencia y continuidad con una tendencia de fondo: el organismo autónomo gestor ha ido incorporando cada vez más competencias siguiendo esta estratégica culturalista en términos de la relevancia concedida a la cultura y de la necesidad

11. Los datos presupuestarios que manejamos proceden de diferentes fuentes: en el caso del Departamento de Cultura de la Generalitat, de sus Memorias anuales (Departament de Cultura, 2011) y de las Estadisticas Culturales (Departament de Cultura, 2012). En el caso de la Diputación de Barcelona, de las Memorias de la institución (Diputació de Barcelona, 2010), y en el caso del Ayuntamiento de Barcelona, del Anuario Estadistico (Ajuntament de Barcelona, 2011). 
de impregnar la política cultural. Por ello, a partir de su creación a mediados de los años noventa, ha incorporado las diversas competencias culturales del Ayuntamiento que en muchos otros Gobiernos locales conforman concejalías autónomas, como son las fiestas y patrimonio (incorporadas el 1995), los proyectos estratégicos culturales de ciudad (Fórum de las Culturas 2004 o la candidatura a Ciudad de la Literatura en 2012-2016) y desde 2011 la gestión del importante programa de Centros Cívicos (57 centros en toda la ciudad). En todo caso, de 1997 a 2011 el organigrama se reorganiza en diversas ocasiones, pero sigue en esencia respondiendo a la idea de una gestión integral, desde la cultura patrimonial a la creación contemporánea; de la cultura de la proximidad y festiva a las grandes instituciones culturales. De hecho, la entrada del equipo de CiU no comportará cambios sustantivos, con la única excepción de la creación de una Dirección de Creatividad e Innovación, siguiendo la lógica de la ciudad creativa coherente con una perspectiva emprendedora que comparten socialdemocracia y derecha liberal acerca de la cultura (Hesmondhalgh et al., 2015). Con el cambio de Gobierno en 2015 se barajó la posibilidad de dividir en dos la gestión cultural: un ámbito que reuniera la gestión de promoción cultural y los grandes equipamientos culturales, y otro ámbito orientado a la participación cultural, más próximo al programa del nuevo equipo. Sin embargo, esta división fue duramente contestada por algunos sectores del ámbito cultural y finalmente se descartó.

Hay que decir que durante este último periodo se ha abandonado la política de los grandes eventos, desarrollada durante veinte años (Rius-Ulldemolins y Sánchez, 2015), con la renuncia a construir en Monjtuïc un nuevo museo de Arquitectura que formara la explanada de los museos, y también se ha llevado a cabo una mayor transparencia en los concursos públicos y las subvenciones, que son publicadas íntegramente (Observatori dades culturals, 2017). No obstante, en global, hasta el momento ninguno de los equipos de gobierno - los comunes o el Gobierno socialista - serán recordados por sus realizaciones, al menos no de momento, aunque la ruptura del pacto de Gobierno entre comunes y socialistas y el nombramiento en 2018 del académico Joan Subirats como Comisionado de la Cultura y su programa de «politización de la cultura» y la potenciación de las redes de cultura de proximidad, abre un interrogante a un año de las elecciones de 2019 sobre la posibilidad de un nuevo giro en la política cultural local (Cervantes, 2018). Sin embargo, actualmente este mandato será más recordado por las batallas culturales en las que se han visto inmersos - o han provocado para tapar la ausencia de actuaciones según el punto de vista que se tome- en relación con cuestiones de debate de representaciones simbólicas (como la exhibición de la estatua de Franco o bien el envío de libros a Trump). Sin embargo, no se puede olvidar que uno de los mayores éxitos culturales de la ciudad, la proclamación por la UNESCO como miembro de la Red Europea de Ciudades Creativas, ha sido una iniciativa surgida de la Universidad de Barcelona, iniciada en el mandato convergente, continuada por los comunes y otorgada otra vez durante el breve mandato socialista (Ajuntament de Barcelona, 2015; Patricio Mulero y Rius-Ulldemolins, 2017). 


\section{CONCLUSIONES}

La crisis económica y financiera ha tenido un impacto muy grande en muchos ámbitos de las sociedades avanzadas y, especialmente, en la esfera cultural. A las incertidumbres de los procesos de digitalización de los sectores culturales se ha sumado en Europa una debilitación de la política cultural, que se ha mostrado con toda su crudeza en el sur de Europa con caídas muy pronunciadas en los presupuestos destinados a la misma y con la eliminación de líneas enteras de actuación pública en cultura (Rubio Arostegui et al., 2014). Asimismo, el optimismo del paradigma de la ciudad creativa, basado en la idea de los efectos benéficos de la cultura para la planificación urbana, el desarrollo social y la cohesión local, ha dejado un rastro de proyectos fallidos, despilfarro de dinero público y barrios gentrificados (Rius-Ulldemolins et al., 2017). Todo ello ha reforzado la diagnosis del agotamiento de los paradigmas clásicos de la política cultural y ha contribuido a la idea de la necesidad de repensar la metasy legitimidad de la política cultural (McGuigan, 2004; Rubio y Rius-Ulldemolins, 2015).

En este contexto, hemos visto emerger con fuerza una corriente, especialmente en las grandes ciudades del Estado español, que ha tendido a reconceptualizar la política cultural como un espacio común y una herramienta de transformación social. A partir de 2015, esta corriente cristalizó en candidaturas de los comunes que alcanzaron las alcaldías de ciudades como Madrid y Barcelona, donde parecía que se abría un profundo ímpetu de cambio que transformaría la relación entre cultura, ciudad y ciudadanía. Sin embargo, dos años después del inicio de esta etapa, el balance es bastante pobre: los presupuestos en ambas ciudades muestran una gran continuidad en sus líneas maestras, una permanencia del gasto principalmente orientado a las grandes organizaciones e instituciones de alta cultura y aumentos moderados de gasto en acción cultural de proximidad. La gestión cultural también sigue adoptando a grandes rasgos formas muy parecidas de externalización en entes públicos de difícil control por parte de los responsables políticos y con escasa participación ciudadana. Finalmente, las experiencias en la introducción de autogestión o gestión comunitaria han sido muy secundarias y no han afectado al núcleo de la política cultural, que sigue desarrollándose en buena medida según los paradigmas anteriores. En ambos casos se observa el triunfo de la inercia sobre el cambio. Por tanto, era necesario analizar por qué las políticas culturales no han cambiado, o no lo han hecho en concordancia con las ambiciosas intenciones de los nuevos responsables locales.

La respuesta a los límites del cambio de las políticas culturales tal como podemos ver en la tabla 1 no es sencilla y unívoca: en ambos casos podemos encontrar factores externos como el corsé que impone el paradigma de la ciudad creativa en sus usos como parte de la marca de la ciudad, en el turismo o bien por la mayor presencia y capacidad de lobby de las industrias creativas hacia la gobernanza local. Por otra parte, la cultura se ha convertido en un elemento destacado en las guerras culturales que los adversarios de estos Gobiernos han utilizado contra los nuevos responsables de las políticas culturales, forzando una cierta parálisis de sus proyectos de cambio. Sin embargo, no debemos menospreciar los efectos de los factores internos en el cambio: 
TABLA I.

COMPARACIÓN DE LA SITUACIÓN DE PARTIDA Y DESARROLLO DE LA POLÍTICA CULTURAL. FACTORES EXÓGENOS Y ENDÓGENOS COMO LIMITACIONES AL CAMBIO (2OI4-20I8)

\begin{tabular}{|c|c|c|}
\hline & Madrid & Barcelona \\
\hline $\begin{array}{l}\text { Situación de } \\
\text { partida }\end{array}$ & $\begin{array}{l}\text { Inicialmente la política cultural no es } \\
\text { un sector relevante en la agenda local. } \\
\text { Se destaca el peso del Ministerio y las } \\
\text { instituciones estatales. Iniciativas de } \\
\text { autogestión cultural que parten o son } \\
\text { animadas por los movimientos } \\
\text { sociales. }\end{array}$ & $\begin{array}{l}\text { Centralidad de la cultural en el } \\
\text { modelo Barcelona. Liderazgo del } \\
\text { Ayuntamiento. Articulación y } \\
\text { rivalidad entre administraciones. } \\
\text { Ausencia de participación de los } \\
\text { movimientos sociales en la gestión } \\
\text { cultural. }\end{array}$ \\
\hline $\begin{array}{l}\text { El cambio } \\
\text { en la } \\
\text { política } \\
\text { cultural }\end{array}$ & $\begin{array}{l}\text { El programa de Ahora Madrid parte } \\
\text { de una crítica al neoliberalismo del } \\
\text { anterior modelo y la fusión entre } \\
\text { cultura y promoción económica y } \\
\text { turística. Parte de las experiencias } \\
\text { autogestionarias como modelo que } \\
\text { extender y una voluntad de } \\
\text { politización de la política cultural. }\end{array}$ & $\begin{array}{l}\text { El programa de Barcelona en Comú } \\
\text { parte de una crítica al paradigma } \\
\text { emprendedor y se propone fomentar } \\
\text { la cultura común y abandonar la } \\
\text { instrumentalización económica y } \\
\text { turística de la cultura. }\end{array}$ \\
\hline $\begin{array}{l}\text { Obstáculos } \\
\text { exógenos al } \\
\text { cambio }\end{array}$ & $\begin{array}{l}\text { Inestabilidad y fricciones en Gobierno } \\
\text { de coalición. Batallas culturales con la } \\
\text { oposición y la prensa. Polémicas en } \\
\text { relación con la libertad de expresión, } \\
\text { dimisiones forzadas. Enfrentamiento } \\
\text { con el sector de las artes escénicas. }\end{array}$ & $\begin{array}{l}\text { Rivalidad entre Barcelona en Comú y } \\
\text { el PSC por el control del área de } \\
\text { cultura. Inestabilidad política y de los } \\
\text { equipos directivos. Batallas culturales } \\
\text { en torno a elementos identitarios. }\end{array}$ \\
\hline $\begin{array}{l}\text { Obstáculos } \\
\text { endógenos } \\
\text { al cambio }\end{array}$ & $\begin{array}{l}\text { Dificultad en el desmontaje de las } \\
\text { estructuras empresariales de gestión } \\
\text { como Madrid Destino. Avances } \\
\text { lentos y modestos en las políticas de } \\
\text { descentralización y democratización } \\
\text { de las políticas culturales. Ausencia de } \\
\text { avances en autogestión. }\end{array}$ & $\begin{array}{l}\text { Inercia en la organización de las } \\
\text { políticas culturales por el ente gestor } \\
\text { (ICUB) y las grandes instituciones } \\
\text { culturales. Dificultad en traducir } \\
\text { operativamente las orientaciones } \\
\text { políticas procomún. }\end{array}$ \\
\hline
\end{tabular}

Fuente: elaboración propia.

la transformación de los objetivos de una política cultural pasa necesariamente por la transformación también de los organigramas y presupuestos, y en este punto estos nuevos Gobiernos se han enfrentado a grandes organismos producto de fusiones anteriores que imponen una complejidad e inercia en la gestión cultural pública, y que no han sabido redimensionar y reenfocar hacia sus objetivos de favorecer una mayor participación ciudadana. Los discursos críticos hacia la ciudad creativa y por la creación de espacios procomún, por innovadores y potencialmente transformadores que fueran, no han ofrecido una alternativa a las lógicas de la promoción de la alta cultura, a la promoción de las industrias culturales o la lógica de la redistribución y difusión de 
la política cultural anterior. Así, el énfasis en la política cultural procomún como un tercer espacio entre el sector público y el privado bajo fórmulas de autogestión ha servido para poner en crisis los esquemas tradicionales de gestión pública de la cultura (Barbieri, 2018), pero ha sido poco útil para reenfocar todo el sistema de la política cultural, más allá de un énfasis en las herramientas clásicas de la democracia cultural (centros culturales y bibliotecas) y de fomentar a experiencias —innovadoras, pero relativamente aisladas - de participación social en la gestión cultural.

Por último, no podemos acabar sin destacar la necesidad de continuar analizando de forma global y longitudinal las políticas culturales locales, no solamente a partir de los discursos y las realizaciones más destacadas (usualmente grandes equipamientos), sino centrándonos en el cambio o continuidad de sus dimensiones estructurales (factores de fondo que explican la organización interna y la distribución presupuestaria del gasto cultural) y sistémicas (relaciones entre agentes). Finalmente, una investigación comparativa de otros grandes municipios — como Valencia, Zaragoza o Santiago de Compostela que, entre otros, conforman los llamados «ayuntamientos del cambio»- ayudaría a reforzar o refutar estos hallazgos sobre el escaso cambio y la continuidad destacable de las políticas culturales locales a corto y medio plazo, a pesar de los cambios de orientación política de los Gobiernos.

\section{Referencias}

Aguilar, Salvador. 2001. Ordre i desordre:manual d'estructura $i$ canvi de les societats. Barcelona: Hacer.

Ajuntament de Barcelona. 1980-2017. Pressupostos de l'Ajuntament de Barcelona. Gaseta Municipal. Barcelona: Ajuntament de Barcelona.

Ajuntament de Barcelona. 2011. Anuari Estadistic de la Ciutat de Barcelona. Barcelona: Ajuntament de Barcelona.

Ajuntament de Barcelona. 2015. Barcelona Ciutat de la Literatura. Candidatura per a la Xarxa de Ciutats Creatives UNESCO. Barcelona: Ajuntament de Barcelona.

Antentas, Josep Maria. 2017. «Podemos and the Spanish political crisis», Labor History, 58: 468-489. Disponible en: https://doi.org/10.1080/0023656X.2017.1255544.

Ariño Villarroya, Antonio. 2010. Prácticas culturales en España :desde los años sesenta hasta la actualidad. Barcelona: Ariel.

Arora, Payal. 2015. «Usurping Public Leisure Space for Protest: Social Activism in the Digital and Material Commons», Space and Culture, 18: 55-68. Disponible en: https://doi.org/10.1177/1206331213517609.

Ayuntamiento de Madrid. 2012. Hacia el Plan Estratégico de Cultura de Madrid 20122015. Madrid: Ayuntamiento de Madrid.

Ayuntamiento de Madrid. 2013. Proyecto de presupuestos 2014. Madrid: Ayuntamiento de Madrid.

Ayuntamiento de Madrid. 2018. Presupuestos Abiertos del Ayuntamiento de Madrid. Madrid: Ayuntamiento de Madrid. 
Barbieri, Nicolás. 2012. "Why does cultural policy change? Policy discourse and policy subsystem: a case study of the evolution of cultural policy in Catalonia», International Journal of Cultural Policy, 18: 13-30. Disponible en: https://doi.org/ 10.1080/10286632.2011.567329.

Barbieri, Nicolás. 2015. «A narrative-interactionst approach to policy change analysis: lessons from a case study of the cultural policy domain in Catalonia», Critical Policy Studies, 9: 434-453. Disponible en: https://doi.org/10.1080/19460171.2015.1014519.

Barbieri, Nicolás. 2018. «Políticas culturales en los ayuntamientos del cambio. ¿Hacia unas políticas públicas de lo común?», Periferica, 18: 183-191.

Barcelona en Comú. 2015. "Gestió pública de la cultura. De la cultura com a recurs a la cutlura com a bé comú", 2es Jornades de l'eix de Cultura Barcelona en Comú, Centre Cívic Casinet d'Hostafrancs.

Barranco, Justo, Maricel Chavaría y Teresa Sesé. 2015. «La cultura de Ada Colau», La Vanguardia, 26/05/2015.

Belfiore, Eleonora. 2009. «On bullshit in cultural policy practice and research: notes from the British case», International Journal of Cultural Policy, 15: 343-359. Disponible en: https://doi.org/10.1080/10286630902806080.

Bouzada, Xan. 2007. «El campo del arte en la génesis de las políticas culturales. Poder y gobernanza en la gestión pública del arte», Politica y Sociedad, 44: 39-55.

Bustamante, Mauricio. 2015. "Les politiques culturelles dans le monde. Comparaisons et circulations de modèles nationaux d'action culturelle dans les années 1980», Actes de la recherche en sciences sociales, 206-207: 156-173. Disponible en: https://doi.org/10.3917/arss.206.0156.

Cervantes, Xavier. 2018. «Joan Subirats: «Hem de polititzar el debat sobre cultura». El nou responsable de l'Icub promou una biennal de cultura i pensament a la tardor», Ara, 25/01/2018.

Connolly, Mark Gerard. 2013. "The "Liverpool model(s)": cultural planning, Liverpool and Capital of Culture 2008", International Journal of Cultural Policy, 19: 162-181. Disponible en : https://doi.org/10.1080/10286632.2011.638982.

Degen, Mónica y Marisol García. 2012. "The Transformation of the Barcelona Model?: An Analysis of Culture, Urban Regeneration and Governance», International Journal of Urban and Regional Research, 36: 1022-1038. Disponible en: https://doi.org/10.1111/j.1468-2427.2012.01152.x.

Departament de Cultura. 2011. Memoria del Departament de Cultura 2010. Barcelona: Departament de Cultura, Generalitat de Catalunya.

Departament de Cultura. 2012. Estadistiques Culturals de Catalunya. Barcelona: Departament de Cultura, Generalitat de Catalunya.

Diputació de Barcelona. 2010. Memòria. Barcelona: Diputació de Barcelona.

Dubois, Vincent. 1999. La politique culturelle. Genèse d'une catégorie d'intervention publique. París: Belin.

Dubois, Vincent. 2016. «The «French model» and its «crisi»: ambitions, ambiguities and challenges of cultural policy», Debats. Journal on Culture, Power and Society, Annual Review 1: 81-97. Disponible en: https://doi.org/10.28939/iam.debats-en.2016-7. 
Evans, Graeme. 2003. «Hard-Branding the cultural city-from Prado to Prada», International Journal of Urban and Regional Research, 27: 417-440. Disponible en: https://doi.org/10.1111/1468-2427.00455.

Gattinger, Monica y Diane St. Pierre. 2010. "The "Neoliberal Turn" in Provincial Cultural Policy and Administration in Québec and Ontario: The Emergence of "Quasi-Neoliberal" Approaches", Canadian Journal of Communication, 35: 279302. Disponible en: https://doi.org/10.22230/cjc.2010v35n2a2185.

Gil, Andrés. 2017. «Manuela Carmena sentencia la concejalía de Cultura: del "omnia sunt communia" al relevo de Celia Mayer», El Diario, 15-03-2017.

Gilmore, Abigail. 2017. «The park and the commons: vernacular spaces for everyday participation and cultural value», Cultural Trends, 26: 34-46. Disponible en: https://doi.org/10.1080/09548963.2017.1274358.

González, Sara. 2011. «Bilbao and Barcelona "in Motion”. How Urban Regeneration "Models" Travel and Mutate in the Global Flows of Policy Tourism", Urban Studies, 48: 1397-1418. Disponible en: https://doi.org/10.1177/0042098010374510.

Gray, Clive. 2004. «Joining-Up or Tagging On? The Arts, Cultural Planning and the View From Below», Public Policy and Administration, 19: 38-49. Disponible en: https://doi.org/10.1177/095207670401900206.

Gray, Clive. 2008. «Instrumental policies: causes, consequences, museums and galleries», Cultural Trends, 17: 209-222. Disponible en: https://doi.org/10.1080/ 09548960802615349.

Gray, Clive. 2009. «Managing cultural policy: pitfalls and prospects», Public Administration, 87: 574-585. Disponible en: https://doi.org/10.1111/j.1467-9299. 2008.01748.x.

Gray, Clive. 2010. «Analysing cultural policy: incorrigibly plural or ontologically incompatible?», International Journal of Cultural Policy, 16: 215-230. Disponible en: https://doi.org/10.1080/10286630902935160.

Gray, Clive y Melvin Wingfield. 2011. «Are governmental culture departments important? An empirical investigation", International Journal of Cultural Policy, 17: 590-604. Disponible en: https://doi.org/10.1080/10286632.2010.549559.

Hesmondhalgh, David, Melissa Nisbett, Kate Oakley y David Lee. 2015. «Were New Labour's cultural policies neo-liberal?», International Journal of Cultural Policy, 21: 97-114.

ICUB. 2017. Organigrama ICUB. Barcelona: Ajuntament de Barcelona.

Landry, Charles y Franco Bianchini. 1995. The creative city. Londres: Demos.

Levi, Simona et al. (ed.). 2012. Tecnopolitica, internet y r-evoluciones. Sobre la centralidad de redes digitales en el \#15M. Barcelona: Icaria.

Marshall, Tim. 2000. «Urban Planning and Governance: Is there a Barcelona Model?», International Planning Studies, 5: 299-319. Disponible en: https://doi. org/10.1080/713672855.

Martí-Costa, Marc y Marc Pradel i Miquel. 2012. «The knowledge city against urban creativity? Artists' workshops and urban regeneration in Barcelona», European 
Urban and Regional Studies, 19: 92-108. Disponible en: https://doi.org/10.1177/ 0969776411422481.

Martínez, Guillem (ed.). 2012. Cultura de la Transición: crítica a 35 años de cultura española. Madrid: Penguin Random House.

McGuigan, Jim. 2004. Rethinking cultural policy. Maidenhead: Open University.

Méndez, Ricardo, Juan J. Michelini, José Prada y Jesús Tébar. 2012. «Economía creativa y desarrolo urbano en España: una aproximación a sus lógicas espaciales», EURE, 38: 5-32. Disponible en: https://doi.org/10.4067/S0250-71612012000100001.

Menger, Pierre-Michel. 1991. "Marché du travail artistique et socialisation du risque : le cas des arts du spectacle», Revue française de sociologie, 32: 61-74. Disponible en: https://doi.org/10.2307/3322356.

Menger, Pierre-Michel. 2005. Les intermittents du spectacle. Sociologie d'une exception. París: Éditions de l'École des hautes études en sciences sociales.

Menger, Piere-Michel. 2010. Cultural policies in Europe. From a state to a city-centered perspective on cultural generativity. Tokyo: National Graduate Institute for Policy Studies.

Menger, Pierre-Michel. 2011. «Les politiques culturelles. Modeles et évolutions», en Philippe Poirrier, (ed.), Pour une histoire des politiques culturelles dans le monde (1945-2011). Paris: La Documentation française.

Ministerio de Educación y Cultura. 2016. Bibliotecas púbicas españolas en cifras. Madrid: Ministerio de Educación y Cultura.

Montañés, Jose Angel. 2017. «Barcelona, el canvi que no va ser. El projecte de Barcelona en Comú, passat al cap d'un any a un regidor del PSC, no ha complert les expectatives», El País, 22-05-2017.

Moulin, Raymonde. 1997. L'Artiste, l'institution et le marché. París: Flammarion.

Murray, Catherine y Jan Marontate. 2010. «Neoliberalism in Provincial Cultural Policy Narratives: Perspectives from Two Coasts», Canadian Journal of Communication, 35: 325-343. Disponible en: https://doi.org/10.22230/cjc.2010v35n2a2183.

Nisbet, Robert y Thomas S. Kuhn. 1993. Cambio social. Madrid: Alianza Universidad.

Observatori dades culturals. 2017. Subvencions i transferències econòmiques de l'ICUB. Barcelona: Ajuntament de Barcelona.

Patricio Mulero, Maria y Joaquim Rius-Ulldemolins. 2017. «From creative city to generative governance of the cultural policy system? The case of Barcelona's candidature as UNESCO City of Literature», City, Culture and Society, 10: 1-10. Disponible en: https://doi.org/10.1016/j.ccs.2017.05.001.

Peck, Jamie. 2005. "Struggling with the Creative Class», International Journal of Urban and Regional Research, 29:740-770. Disponible en: https://doi.org/10.1111/ j.1468-2427.2005.00620.x.

Ramoneda, Josep (ed.). 1997. Barcelona Madrid 1898-1998. Barcelona: Centre de Cultura Contemporània de Barcelona.

Rius-Ulldemolins, Joaquim. 2006. «El MACBA i el CCCB. De la regeneració cultural a la governança cultural», Digithum, 8: 10-17.

Rius-Ulldemolins, Joaquim. 2013. «¿Es posible gobernar los teatros de ópera? Análisis del caso del Gran Teatro del Liceu de Barcelona», Revista Española de Ciencia Política, 33: 104 . 
Rius-Ulldemolins, Joaquim. 2014. «Culture and authenticity in urban regeneration processes: Place branding in central Barcelona», Urban Studies, 51: 3026-3045. Disponible en: https://doi.org/10.1177/0042098013515762.

Rius-Ulldemolins, Joaquim. 2014a. «La gobernanza y la gestión de las instituciones culturales nacionales : de la oposición entre arte y economía a la articulación entre política cultural y management», Revista Papers, 99: 73-95. Disponible en: https:// doi.org/10.5565/rev/papers/v99n1.542.

Rius-Ulldemolins, Joaquim. 2014b. «Modelos de política cultural y modelos de equipamientos culturales: de los modelos nacionales a los modelos locales. Análisis del caso de Barcelona", Politica y Sociedad, 51: 399-422.

Rius Ulldemolins, Joaquín, Arturo Rodríguez Morató y Santi Martinez Illa. 2012. «El sistema de la política cultural en Cataluña: un proceso inacabado de articulación y racionalización», Revista de Investigaciones Políticas y Sociológicas, 11:31-173.

Rius-Ulldemolins, Joaquim, Gil-Manuel Hernández i Martí y Francisco Torres Pérez. 2015. «Urban development and cultural policy "white elephants": Barcelona and Valencia", European Planning Studies, 24 (1): 1-21.

Rius-Ulldemolins, Joaquim, Vicent Flor Moreno y Gil Manuel Hernàndez y Martí. 2017. "The dark side of cultural policy: economic and political instrumentalisation, white elephants, and corruption in Valencian cultural institutions", International Journal of Cultural Policy, OnlineFirst: 1-16.

Rius-Ulldemolins, Joaquim y Arturo Rubio. 2013. «The governance of national cultural organisations: comparative study of performance contracts with the main cultural organisations in England, France and Catalonia», International Journal of Cultural Policy, 19: 249-269. Disponible en: https://doi.org/10.1080/10286632.2011.638981.

Rius-Uldemollins, Joaquim y Mariano Martín Zamorano. 2014. «¿Es España un estado casi-federal en política cultural? Articulación y conflicto entre la política cultural de la Administración General del Estado y de la Comunidad Autónoma de Cataluña", Revista d'Estudis Autonòmics i Federals., 19: 274-309.

Rius Ulldemolins, Joaquim y Mariano Martín Zamorano. 2015. «Spain's nation branding project Marca España and its cultural policy: The economic and political instrumentalization of a homogeneous and simplified cultural image», International Journal of Cultural Policy, 21: 20-40.

Rius-Ulldemolins, Joaquim y María Victoria Sánchez. 2015. «Modelo Barcelona y política cultural: usos y abusos de la cultura por parte de un modelo emprendedor de desarrollo local», EURE, 41: 101-123.

Rius-Ulldemolins, Joaquim y Santi Martinez Illa 2016. «El sistema de la política cultural del Estado de las autonomías: entre la articulación, la concurrencia y el clientelismo», en Joaquim Rius Ulldemolins y Juan Arturo Rubio Arostegui (eds.), Treinta años de politicas culturales en España. Participación cultural, gobernanza territorial e industrias culturales. València: Publicacions de la Universitat de València.

Rosi, Mauro. 2014. «Branding or sharing?: The dialectics of labeling and cooperation in the UNESCO Creative Cities Network», City, Culture and Society, 5: 107-110. Disponible en: https://doi.org/10.1016/j.ccs.2014.05.002. 
Rowan, Jaron. 2015. «Madrid Destino es un sistema vertical en toda regla, que trabaja más cerca de los intereses del mercado", El Diario, 26-07-2015.

Rubio Arostegui, Juan Arturo. 2003. La politica cultural del Estado en los gobiernos socialistas: 1982-1996. Gijón: Trea.

Rubio Arostegui, Juan Arturo. 2005. «La política cultural del Estado en los gobiernos populares (1996-2004): entre el ¿liberalismo? y el continuismo socialista", Revista Sistema, 187: 111-124.

Rubio Arostegui, Juan Arturo. 2012. «La política cultural de los Gobiernos autonómicos de la Comunidad de Madrid: su singularidad en el contexto autonómico español», Revista de Investigaciones Politicas y Sociológicas, 11: 205-234.

Rubio Arostegui, Juan Arturo. 2014. Análisis institucional y financiero del sector de las artes escénicas: claves, ejes y bases para un futuro sostenible. Madrid: Mecartes.

Rubio Arostegui, Juan Arturo, Joaquim Rius-Ulldemollins y Santi Martinez Illa 2014. El modelo español de financiación de las artes y la cultura en el contexto europeo. Crisis económica, cambio institucional, gobernanza y valor público de la cultura y la política cultural. Madrid: Fundación Alternativas.

Rubio, Arturo y Joaquim Rius-Ulldemolins. 2015. "Cultura y políticas públicas después del diluvio. Las ciencias sociales y la refundación de la política cultural», Politica y Sociedad, 51: 27-52.

Saint-Pierre, Diane. 2003. La politique culturelle du Québec de 1992: Continuité ou changement. Les acteurs, les coalitions, les enjeux. Québec: Les Presses de l'Université Laval.

Sánchez Belando, Victoria y Joaquim Rius-Ulldemolins. 2014. "The creative city’s cracks: contestation and social innovation in the city of Barcelona. The Case of the Socio-Cultural Center Can Batlló", 8th International Conference on Cultural Policy Research, Septiembre 9-12, 2014.

Sánchez, María Victoria, Joaquim Rius-Ulldemolins y Matías Zarlenga. 2013. «¿Ciudad creativa y ciudad sostenible?: un análisis crítico del modelo Barcelona de políticas culturales", Revista Critica de Ciências Sociais, 96: 48-57.

Scott, Allen. 2010. "Cultural economy and the creative field of the city», Geografiska Annaler: Series B, Human Geography, 92: 115-130. Disponible en: https://doi. org/10.1111/j.1468-0467.2010.00337.x.

Shockley, Gordon E. 2011. «Political Environment and Policy Change: The National Endowment for the Arts in the 1990s", The Journal of Arts Management, Law, and Society, 41: 267-284. Disponible en: https://doi.org/10.1080/10632921.2011.62 8232.

Sola, Jorge y Cé Rendueles. 2018. «Podemos, the upheaval of Spanish politics and the challenge of populism», Journal of Contemporary European Studies, 26 (1): 99-116.

Subirós, Josep. 1999. Estrategias culturales y renovación urbana. Barcelona: Aula Barcelona.

Sztompka, Piotr. 1993. The Sociology of Social Change. Hoboken, NJ: Wiley-Blackwell.

Treballadors/es independents de la cultura. 2016. Volem que la politica cultural a Barcelona ciutat canviï. Barcelona: Change. 
Urfalino, Philippe. 1984. Le jeu du catalogue. Les contraintes de l'action culturelle dans les villes. París: La Documentation Française.

Urfalino, Philippe. 1996. L'invention de la politique culturelle. París: La Documentation Française.

Vos, Claske. 2017. «European integration through "soft conditionality". The contribution of culture to EU enlargement in Southeast Europe», International Journal of Cultural Policy, 23: 675-689. Disponible en: https://doi.org/10.1080/1028663 2.2016.1276577.

Williams, S. y Elizabeth Currid-Halkett. 2011. «The emergence of Los Angeles as a fashion hub: A comparative spatial analysis of the New York and Los Angeles fashion industries», Urban Studies, 48: 3043-3066. Disponible en: https://doi. org/10.1177/0042098010392080.

Zimmer, Annette y Stefan Toepler. 1996. «Cultural Policies and the Welfare State: The Cases of Sweden, Germany, and the United States», The Journal of Arts Management, Law and Society, 26: 167-193. Disponible en: https://doi.org/10.1080/10 632921.1996 .9942961$.

Presentado para evaluación: 22 de septiembre de 2017. Aceptado para publicación: 19 de abril de 2018.

\section{JOAQUIM RIUS-ULLDEMOLINS}

joaquin.rius@uv.es

Doctor en Sociología por la Universidad Autónoma de Barcelona y la École des Hautes Études en Sciences Sociales. Actualmente, es profesor ayudante doctor en el Departamento de Sociología y Antropología Social de la Universidad de Valencia. Es autor de varios libros y artículos sobre sociología de la cultura y política cultural en revistas nacionales e internacionales como International Journal of Cultural Policy, Urban Studies, European Urban and Regional Studies, Revista Española de Investigaciones Sociológicas y Revista Internacional de Sociología, entre otras.

\section{VERÓNICA GISBERT}

\section{veronica.gisbert@uv.es}

Candidata al doctorado y profesora asociada del Departamento de Sociología y Antropología Social de la Universidad de Valencia. Licenciada en Criminología y máster Erasmus Mundus en Estudios sobre la Mujer y el Género. Sus investigaciones se centran en la temática de género y política, derechos humanos y procesos de conflicto. Es autora de varios trabajos sobre política cultural, el movimiento feminista y las festividades tradicionales. 